\title{
DETERMINATION OF PARTICLE SHAPE AND SIZE DISTRIBUTION OF MODEL TYPES OF NANOMATERIALS
}

\author{
Edita Bretšnajdrová ${ }^{*}$ — Ladislav Svoboda * Jiří Zelenka ${ }^{* *}$
}

\begin{abstract}
At present, great attention is given to study of preparation and properties of various nanomaterials usable in many applications. They are utilized in varied fields of human activity - eg in electronics, medicine, paint industry etc. Besides the detailed chemical structure, such nanoparticle properties as the shape and size distribution are fundamental to the given application. To measure these parameters various methods are used, e.g. transmission electron microscopy (TEM), atomic force microscopy (AFM), acoustic spectrometry, methods based on the light scattering and X-ray disc centrifuge system.

K e y w o r d s: particle size distribution, silica, sodium montmorillonite
\end{abstract}

\section{INTRODUCTION}

Acoustic spectrometry, methods based on the light scattering, X-ray disc centrifuge system, AFM and TEM were used in this work to characterize particles of two selected model types of nanomaterials - colloidal silica and sodium montmorillonite. Silica represents a material with spherical particles. Tabular shapes are typical for montmorillonite materials.

Experiments proved that methods used to measure particle size distribution based on the light scattering require very diluted dispersions. But, on the other hand, these conditions can affect the particle size distribution due to agglomeration or deagglomeration of the particles. For these reasons, methods working with concentrated suspensions, without great dilution, are more suitable to get correct results for such colloidal systems, for example acoustic spectrometry and X-ray disc centrifuge system. To obtain information on the shape of particles TEM and AFM methods were used.

Merits and limitations of single methods used to evaluate nanoparticles of various types are discussed in this work.

\section{MATERIALS}

\subsection{Silica}

- Bindzil cc 30, Eka Chemicals AB, Sweden, dispersion of silica nanoparticles, $30 \%$ solution in water, particle size $7 \mathrm{~nm}$, sterically stabilized.

- Bindzil 30/360, Eka Chemicals AB, Sweden, dispersion of silica nanoparticles, $30 \%$ solution in water, particle size $7 \mathrm{~nm}$, electrostatically stabilized.

\subsection{Montmorillonite}

- Cloisite $\mathrm{Na}^{+}$, Southerm Clay Products, Inc., USA, powdery clay, CEC $90 \mathrm{meq} / 100 \mathrm{~g}$.

\section{EXPERIMENTAL}

\subsection{Ultrasound spectroscopy}

The ultrasound based technique is suitable for characterizing heterogeneous solid in liquid or liquid in liquid colloidal systems. This method is suitable for concentrated solutions; it is reliable in the range from $5 \%$ to $50 \%$. We have used equipment DT-1200 (Dispersion technology, USA). The range of measurement of particle size is from $0.005 \mu \mathrm{m}$ to $1000 \mu \mathrm{m}$ and frequency range is from 1 to $100 \mathrm{MHz}$.

\subsection{Dynamic light scattering}

The technique of laser diffraction is based on the principle that particles passing through a laser beam will scatter light at an angle that is directly related to their size. Particle size distribution measurement was carried out by dynamic light scattering using two types of instruments - Mastersizer $2000 \mathrm{MU}$ and 90 Plus/BI-MAS.

Matersizer 2000 MU (Malvern, United Kingdom) measures materials from $0.02 \mu \mathrm{m}$ to $2000 \mu \mathrm{m}$. This instrument is suitable for the measurement of emulsion, suspension and dry powders.

90 Plus/BI-MAS (Brookhaven Instruments Corporation, USA) is an automatic particle sizer designed for used with either concentrated suspensions of small particles or solutions of macromolecules. Sizes from $2 \mathrm{~nm}$ to $3 \mu \mathrm{m}$ can be measured.

\footnotetext{
* University of Pardubice, Faculty of Chemical Technology, Department of Inorganic Technology, Nám(e)stí Československých Legií 565, 53210 Pardubice, Czech Republic, ${ }^{* *}$ Synpo, akciová společnost, S.K. Neumanna 1316, 53207 Pardubice, Czech Republic, edita.bretsnajdrova@student.upce.cz
} 


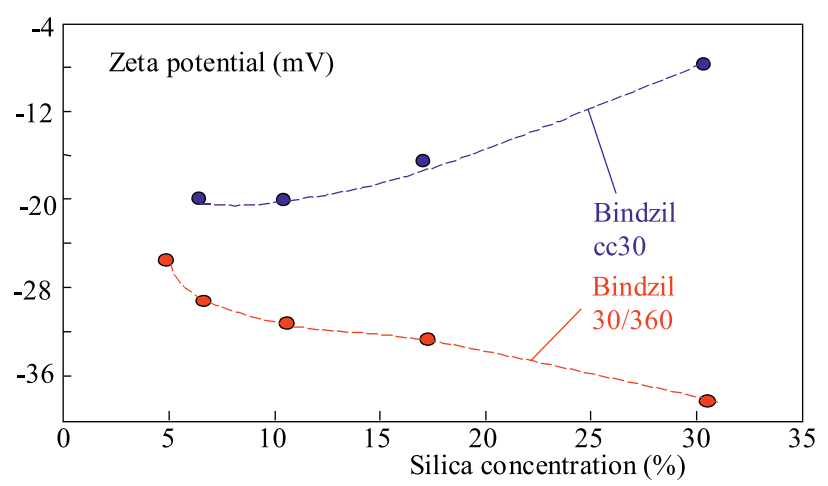

Fig. 1. Dependence of zeta potential on silica concentration

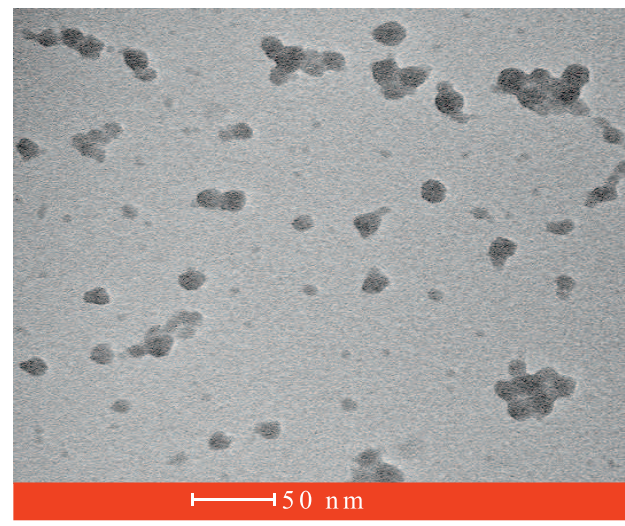

Fig. 3. TEM photo of original sample Bindzil cc 30

\subsection{X-ray disc centrifuge system}

For measurement of particle size the X-ray disc centrifuge system (Brookhaven Instruments Corporation, USA) was used. XDC method allows to make measurements in either a centrifugal or a gravitational field. Xrays from a low power X-ray tube are passed through the disc. The intensity of the beam is attenuated in proportion to the concentration of the suspension through which it passes. The intensity of the transmitted beam is measured with a detector consisting of a scintillation counter whose output is recorded by the computer as a function of time. For a homogeneous suspension, the attenuation of the X-ray beam is proportional to the mass concentration of the suspension at the measurement radius.

\subsection{AFM}

A Solver Pro M Atomic Force Microscope (NT-MDT, Russia) was used in tapping mode (semi-contact) to produce three-dimensional images of the surface. High resolution "golden silicon cantilever NSG-10 (Au coating, curvature radius $10 \mathrm{~nm}$ and cone angle less than $22^{\circ}$ ) were used for all measurements. Set point was adjusted on $50 \%$ of a free oscillation. Scan sizes required to evaluate the distribution of particles was $500 \times 500 \mathrm{~nm}$ to several microns depending on variation of particles size.

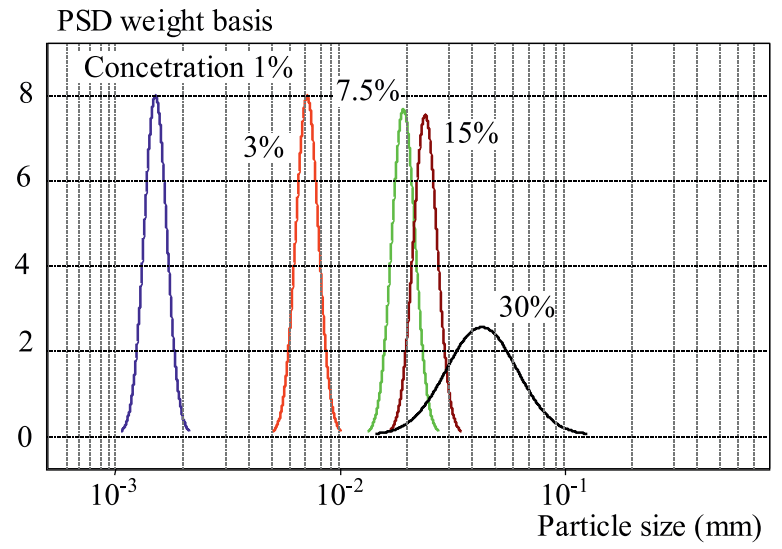

Fig. 2. Particle size distribution on silica concentration

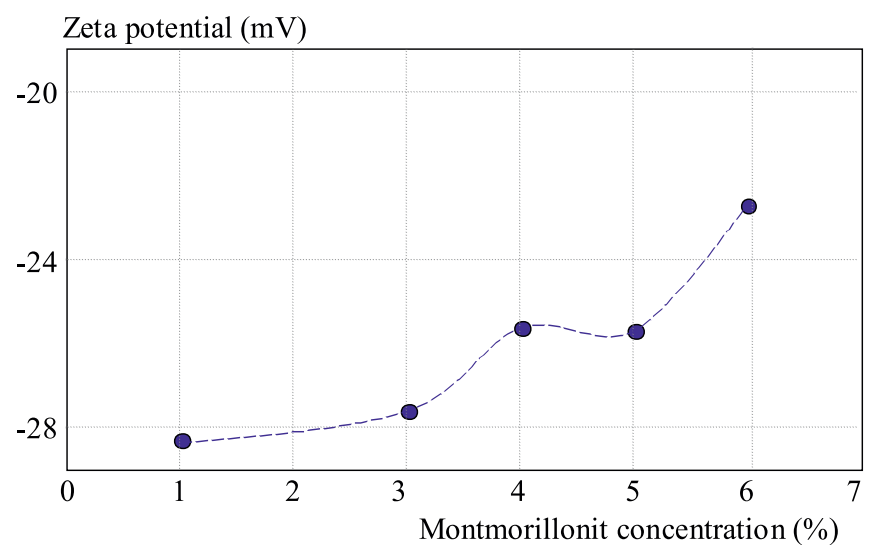

Fig. 4. Dependence of zeta potential on montmorillonite concen-

\subsection{TEM} tration

A drop of water-diluted suspension was put on a microscopic grid covered by ultra thin carbon film and observed directly with TEM Tecnai G2 Spirit Twin (FEI, USA).

\section{RESULTS}

\subsection{Silicas}

Two types of commercial silicas were tested (Bindzil cc 30 and Bindzil 30/360). These silicas are stabilized in different ways. While Bindzil cc 30 is sterically stabilized, Bindzil 30/360 is stabilized electrostatically. The effect of silica concentration on zeta potential and particle size was studied for both type of stabilization. The variety of silica concentrations were prepared by dilution of original samples. The type of stabilization has crucial effect on silica concentration dependence of the zeta potential (Fig. 1).

The concentration of silica has an effect on the particle size and particle size distribution. An example for Bindzil cc 30 is in Fig. 2.

The original sample of Bindzil cc 30 has relatively broad particle size distribution. The mean value of the particle size is about $40 \mathrm{~nm}$. The measured value is higher than the value cited by the producer. The presence of 

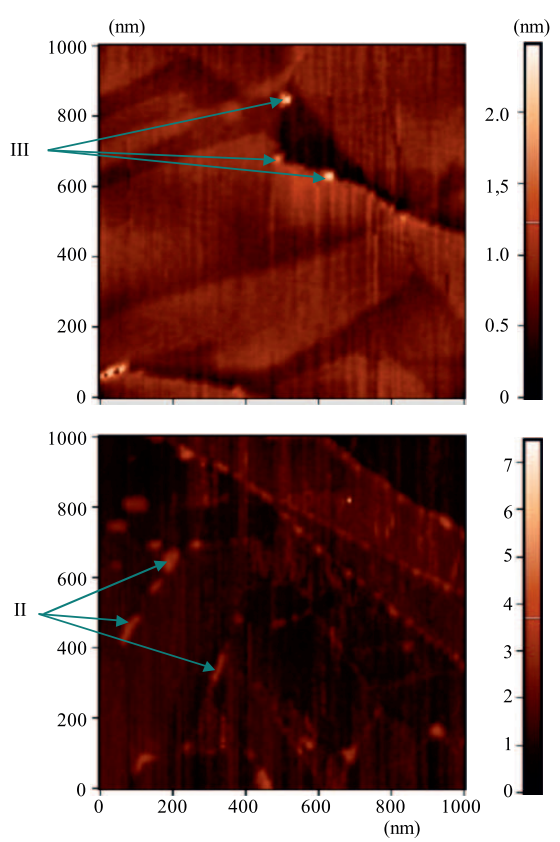

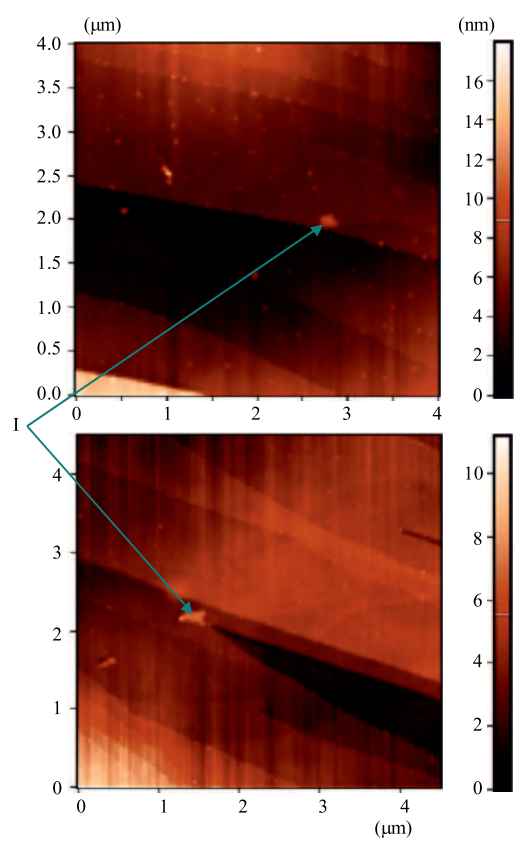

Fig. 5. AFM photo of sodium montmorillonite

bigger formations was confirmed by transmission electron microscopy (Fig. 3).

\subsection{Montmorilonitte}

The zeta potential of water-based dispersion of sodium montmorillonite was influenced by filler concentration (Fig. 4).

The particles with different shapes and dimensions were observed by means of different techniques (TEM, AFM). Results from AFM are in Fig. 5.

Pentagon particles with particle size about $200 \mathrm{~nm}$ can be observed in section I of Fig. 5. Lengthwise formation with highest dimension $100 \mathrm{~nm}$ can be seen in part II of Fig. 5. Round particles with diameter $30 \mathrm{~nm}$ are in part III. The thickness of all studied particles is practically the same - about $1 \mathrm{~nm}$.

\section{CONCLUSIONS}

The type of silica particle stabilization influenced behaviour of colloidal solutions during dilution (particle size and zeta potential changes). All used methods give similar results. Bigger particles (aggregates primary particles) were observed by means of AFM and TEM. These aggregates were broken up by dilution of original samples.

Commercial sodium montmorillonite is compound of elements with different shapes and sizes. There are lengthwise formations, circular and pentagonal particles.

\section{Acknowledgements}

This work has been supported by the projects FTTA3/055 and FT-TA4/074 of the Ministry of Industry and Trade of the Czech Republic and has been supported from the resources of the research intention MSM 0021627501 .

\section{REFERENCES}

[1] KOSMUlSKI, M.-DAHLSTEN, P.: Colloids and Surfaces 291 (2006), 212-218.

[2] PIERRE, A. C.: Journal of Materials Science 32 (1997), 2937-2947.

[3] YUKSELEN, Y.-KAYA, A.: Water, Air and Soil Pollution 145 (2003), 155-168.

Received 30 June 2010

Edita Bretšnajdrová was born in Hořice, Czech Republic, in 1983. She received MS degree from University of Pardubice. At present she is $\mathrm{PhD}$ student at the University of Pardubice, Faculty of Chemical Technology. Her research interests are preparation and properties evaluation of inorganic nanoparticles.

Ladislav Svoboda was born in Pardubice, Czech Republic, in 1954. He received MS and $\mathrm{PhD}$ degree from the University of Pardubice. At present he is Head of Department of Inorganic Technology at University of Pardubice. His research interests are studies of fertilisers with controlled release of nutrients and development of methods for inorganic analysis.

Jiří Zelenka was born in Pardubice, Czech Republic, in 1955. He received MS degree from the University of Pardubice and $\mathrm{PhD}$ degree from Institute of Macromolecular Chemistry of the Academy of Sciences of the Czech Republic. At present he is Head of Department of Nanostructured polymers and renewable raw materials in Synpo company. His research interests are preparation and surface modification of nanoparticles for various selected polymer binder and application, physical evaluation of polymers and polymer composite.

This work was presented at Nanoved \& Nanotech \& Techtransfer 2010, International Conference on Nanoscience, Nanotechnology, Nanomaterials, Nanomedicine and Transfer 1619 May 2010, Bratislava, Slovakia. 